\title{
Radiation Coordinates of Florides - McCrea - Synge
}

\author{
J. López-Bonilla ${ }^{1}$, R. López-Vázquez ${ }^{1}$, G. R. Pérez-Teruel ${ }^{2}$ \\ ${ }^{I}$ ESIME-Zacatenco, Instituto Politécnico Nacional, \\ ${ }^{I}$ Edif. 5, 1er. Piso, Col. Lindavista CP 07738 México DF \\ ${ }^{2}$ Depto. de Física Teórica, Univ. de Valencia, Burjassot-46 100, Valencia, Spain \\ Corresponding author: jlopezb@ipn.mx
}

\begin{abstract}
In this work we construct the element of volume vector $d \sigma_{r}$ of a surface of constant retarded distance around the trajectory of a charged particle with arbitrary motion in a Riemannian space. This constitutes a generalization of the method pioneered by Synge [1] in special relativity. The technique employed is suggested by the 'radiation coordinates' $y^{r}$ introduced by Florides-McCrea-Synge [2,3] in the study of gravitational radiation.
\end{abstract}

Keywords: Radiation Coordinates, Surfaces of Constant Retarded Distance

\section{Introduction}

Here, the Florides-McCrea-Synge coordinates [2,3] are used for the electromagnetic radiation and are considerably adapted to this purpose because, for such coordinates, the curved space behaves like a "flat space" in some aspects. That is, the use of $y^{r}$ implies that what was learned in Minkowski space can be naturally translated to a Riemannian space. Our expression for the element of volume vector $d \sigma_{r}$, of a surface of constant retarded distance, agrees with that obtained by Villarroel [4] by means of the procedure that DeWitt-Brehme [5] use when constructing a surface with constant instantaneous distance. However, we think that our method is simpler and more powerful, because it turns immediate the results on radiation tensors deduced in [6]. We shall use the World Function $\Omega$ of Ruse [7] which allows having covariant expansions in a curved space. This function remained forgotten for a long time, and its present relevance may be seen in [5, 8-20].

\section{Radiation Coordinates}

We assume the Dedekind (1868) [21, 22]-Einstein summation convention for the addition of repeated indices, and that the metric locally takes the form, $\left(\eta_{a b}\right)=(1,1,1,-1)$ at any event. In order to construct the radiation coordinates $y^{r}$ [2] we need a timelike curve $C$ (which in this case will be the electron trajectory) with an orthonormal tetrad on it:

$$
\lambda(a)_{i^{\prime}} \lambda(b)^{i^{\prime}}=\eta_{a b}, \quad \lambda(a) i^{\prime} \lambda^{(a)} j^{\prime}=g_{i^{\prime} j^{\prime}}, \quad \lambda(a)^{i^{\prime}}=\lambda^{i^{\prime}}
$$


where, $\lambda^{i^{\prime}}=\frac{d x^{i^{i}}}{d s}$ is the unitary tangent vector to $C$, and $x^{r}$ is a totally arbitrary coordinate system with $d s^{2}=g_{i j} \cdot d x^{i} \cdot d x^{j}$. The primed indices label points on $C$. Now let us see how $x^{r}$ gives new coordinates: We parameterize the null geodesic $P^{\prime} P$ in the form $x^{r}(v)$ with

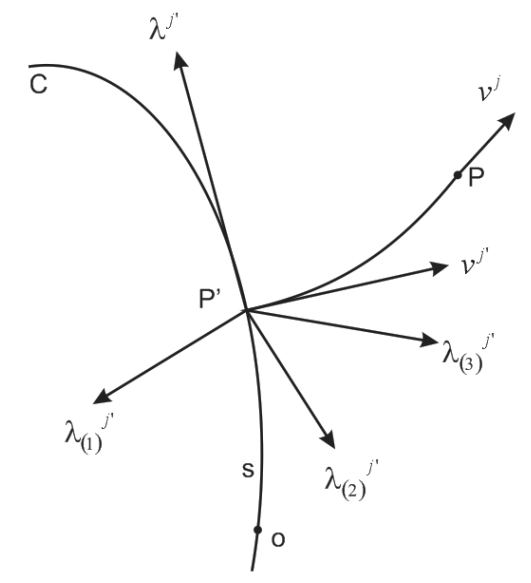

Fig. 1. For every $P$ we construct the past sheet of its null cone which intersects to $C$ in $P$ ' (retarded point associated to $P$ ).

$v=v_{0}$ at $P^{\prime}$, and $v=v_{1}>v_{0}$ at $P$ with $V^{r}=\frac{d x^{r}}{d v}$ as its tangent vector, satisfying $V^{r} V_{r}=0$. The assigned radiation coordinates to $P$ are given by:

$$
y^{r}=-\Omega_{j^{\prime}} \lambda^{(r) j^{\prime}}+s \lambda_{j^{\prime}} \lambda(r) j^{\prime}
$$

where $\Omega_{j^{\prime}}$ denote the covariant derivative of $\Omega$, see Synge [14]:

$$
\Omega_{j^{\prime}}=-\left(v_{1}-v_{0}\right) V j^{\prime}, \quad \Omega_{j^{\prime}} \Omega^{j^{\prime}}=0,
$$

so that $y^{\sigma}=-\Omega_{j^{\prime}} \lambda^{(\sigma) j^{\prime}}, \quad y^{4}=\Omega_{j^{\prime}} \lambda^{j^{\prime}}+s$ which implies that in radiation coordinates the curve $C$ is reduced to $y^{\sigma^{\prime}}=0, y^{4^{\prime}}=s$. If we introduce the notation:

$$
\xi_{j^{\prime}}=-\Omega_{j^{\prime}}, \quad w=-\xi_{j^{\prime}} \lambda^{j^{\prime}}=\Omega_{j^{\prime}} \lambda^{j^{\prime}}
$$

then we obtain the form of the relation (9.3) of Synge [1] for flat space:

$$
y^{\sigma}=y_{\sigma}=\xi_{j}, \lambda^{(\sigma) j^{\prime}}, \quad y^{4}=-y_{4}=w+s,
$$

in this sense the curved space behaves like a Minkowski space-time, which is very useful. On the other hand, at $P$ ' the metric tensor can be written in terms of the tetrad as:

$$
g_{i^{\prime} j^{\prime}}=\lambda^{(\sigma) i^{\prime}} \lambda_{(\sigma) j^{\prime}}-\lambda_{i^{\prime}} \lambda_{j^{\prime}}
$$

then $y^{\sigma} y_{\sigma}=\xi_{i^{\prime}} \xi_{j^{\prime}}\left(g^{i^{\prime} j^{\prime}}+\lambda^{i^{\prime}} \lambda^{j^{\prime}}\right)=w^{2}$ due to $(3,4)$, from where $\xi_{j^{\prime}}=y^{\sigma} \lambda_{(\sigma) j^{\prime}}+w \lambda_{j^{\prime}}$, 
therefore $y^{r}-y^{r^{\prime}}$ behaves like a null vector $\left(y^{r}-y^{r^{\prime}}\right)\left(y_{r}-y_{r^{\prime}}\right)=0$. Thus, our expressions are compatibles with $(4,5,9)$ of [1]. Following the corresponding procedure in flat space let us introduce a new system of coordinates:

$$
z^{\sigma}=y^{\sigma}, \quad z^{4}=y^{4}-\sqrt{y^{\sigma} y^{\sigma}}=s,
$$

that is, $z^{4}$ remains constant on the null cone with vertex at $P^{\prime}$. It is clear that the Jacobian of the transformation $\left(y^{r} \longrightarrow z^{r}\right)$ is equal to one, $J\left(z^{i} / y^{r}\right)=\operatorname{det}\left(\partial z^{i} / \partial y^{r}\right)=1$, therefore:

$$
J\left(\frac{z^{a}}{x^{b}}\right)=J\left(\frac{y^{a}}{x^{b}}\right)
$$

now let us calculate (8). We have that $\frac{\partial z^{\sigma}}{\partial x^{i}}=-\Omega_{i i} \lambda^{(\sigma) i^{i}}+N^{\sigma} \Omega_{i}, \quad \frac{\partial z^{4}}{\partial x^{i}}=-w^{-1} \Omega_{i}$ with $N^{\sigma}=w^{-1}\left(\Omega_{i^{\prime} j^{\prime}} \lambda^{r^{\prime}} \lambda^{(\sigma) j^{\prime}}+\Omega_{r^{\prime}} \frac{d}{d s} \lambda^{(\sigma) r^{\prime}}+\Omega_{r^{\prime}} \frac{d}{d s} \lambda^{(\sigma) r^{\prime}}\right)$, where were employed the properties $\frac{\partial x^{r^{\prime}}}{\partial x^{r}}=\lambda^{r^{\prime}} s_{, r}=-w^{-1} \lambda^{r^{\prime}} \Omega_{r}, \quad \Omega_{r}=\left(v_{1}-v_{0}\right) V_{r}$, hence:

$$
J\left(\frac{z^{a}}{x^{b}}\right)=\varepsilon^{i j k m} \frac{\partial z^{1}}{\partial x^{i}} \frac{\partial z^{2}}{\partial x^{j}} \frac{\partial z^{3}}{\partial x^{k}} \frac{\partial z^{4}}{\partial x^{m}}=w^{-1} \mathcal{E}^{i k k m} \Omega_{j^{\prime} i} \Omega_{r^{\prime} j} \Omega_{t^{\prime} k} \Omega_{m} \lambda^{(1) j^{\prime}} \lambda^{(2) r^{\prime}} \lambda^{(3) t^{\prime}},
$$

for the skew-symmetric nature of the Levi-Civita density $\varepsilon^{i j k m}$. On the other hand, the World Function satisfies $\Omega_{m}=\Omega_{p^{\prime} m} \Omega^{p^{\prime}}$, substituting this into (9) we get:

$$
J\left(\frac{z^{a}}{x^{b}}\right)=w^{-1} \operatorname{det}\left(-\Omega_{a^{\prime} b}\right) \mathcal{E}_{j^{\prime} r^{\prime} t^{\prime} p^{\prime}}, \lambda^{(1) j^{\prime}} \lambda^{(2) r^{\prime}} \lambda^{(3) t^{\prime}} \Omega^{p^{\prime}} ;
$$

from (3) it is clear that $\Omega^{p^{\prime}}$ can be written in terms of the tetrad:

$$
\Omega^{p^{\prime}}=a_{\sigma} \lambda^{(\sigma) p^{\prime}}+a_{4} \lambda^{p^{\prime}} \quad \therefore \quad w=\Omega_{p^{\prime}} \lambda^{p^{\prime}}=-a_{4},
$$

then, thanks to the skew-symmetry of $\varepsilon^{i j k m}$, equation (10) acquires the form:

$$
J\left(\frac{z^{a}}{x^{b}}\right)=\operatorname{det}\left(-\Omega_{a^{\prime} b}\right) \varepsilon_{j^{\prime} r^{\prime} t^{\prime} p^{\prime}} \lambda^{(1) j^{\prime}} \lambda^{(2) r^{\prime}} \lambda^{(3) t^{\prime}} \lambda^{(4) p^{\prime}}=\operatorname{det}\left(-\Omega_{a^{\prime} b}\right) \operatorname{det}\left(\lambda^{(r) i^{\prime}}\right)=-g^{-1 / 2}\left(p^{\prime}\right) D,
$$

where $D=-\left|-\Omega_{a^{\prime} b}\right|, \quad g\left(P^{\prime}\right)=-\left|g_{i^{\prime} j^{\prime}}\right|$. Let us introduce the notation:

$$
\Delta=\bar{g}^{-1} D=g^{-1 / 2}(P) g^{-1 / 2}\left(P^{\prime}\right) D, \quad g(P)=-\left|g_{i j}\right|,
$$

thus from (11):

$$
J\left(\frac{z^{a}}{x^{b}}\right)=-g^{1 / 2}(P) \Delta
$$


Taking into account the last identity it is clear the remark in [5] page 231 and [10] page 1251: the geodesics emerging from $P$ begin their intersection when $\Delta^{-1}=0$, arising the so-called 'caustic surface'. We shall therefore accept that $P$ is near to $P^{\prime}$, in order to have this only geodesic between them. The analysis performed allows consider the volume element of the curved spacetime:

$$
d^{4} x=\left|J\left(\frac{x}{z}\right)\right| d^{4} z=g^{-1 / 2}(P) \Delta^{-1} d s d^{3} z,
$$

but $z^{\sigma}=w p^{\sigma}=w p_{i} \lambda^{(\sigma) i^{\prime}}$ with $p_{i}=w^{-1} \xi_{i^{\prime}}-\lambda_{i^{\prime}}=$ unitary spacelike vector :

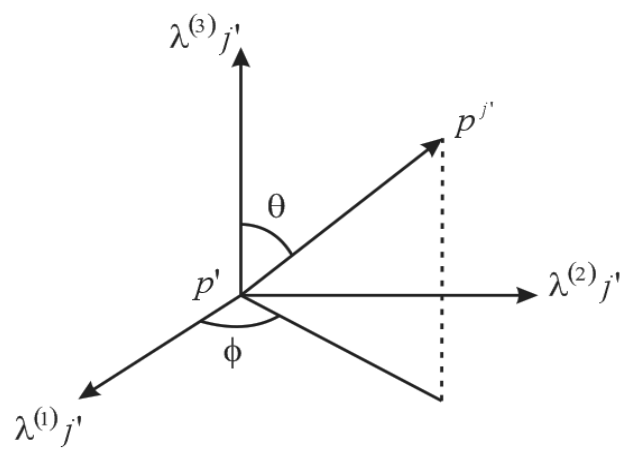

Fig. 2. The quantities $p^{\sigma}$ represent the components of $p^{j^{\prime}}$ in the basis $\lambda^{(\sigma) j^{\prime}}$

Therefore, $\quad z^{1}=w \sin \theta \cos \phi, \quad z^{2}=w \sin \theta \operatorname{sen} \phi, \quad z^{3}=w \cos \theta \quad$ which implies $d^{3} z=w^{2} d w d \gamma$ where $d \gamma=\sin \theta d \theta d \varphi$ is the element of solid angle in the rest frame of the charge. Then (14) adopts the form:

$$
d^{4} x=g^{-1 / 2}(P) \Delta^{-1} w^{2} d s d w d \gamma,
$$

which together with (13) represents the generalization to Riemannian spaces of the results $(9.15$, 21) of Synge [1] (who made use of imaginary coordinates) for Minkowski space-time:

$$
J\left(\frac{z^{a}}{x^{b}}\right)=-1, \quad d^{4} x=w^{2} d s d w d \gamma .
$$

In the next section we will apply (15) to the particular case of the surface $w=$ constant, which is important when studying the electromagnetic radiation

\section{Surface of Constant Retarded Distance}

Let us consider the 3 -space $w=$ constant, then the covariant derivative $w_{; r}$ is orthogonal to that surface. It is therefore evident that its vector volume element is given by (where $d \sigma$ is the 3element of volumen):

$$
d \sigma_{r}=\left|w_{; a} w^{; a}\right|^{-1 / 2} w_{; r} d \sigma
$$




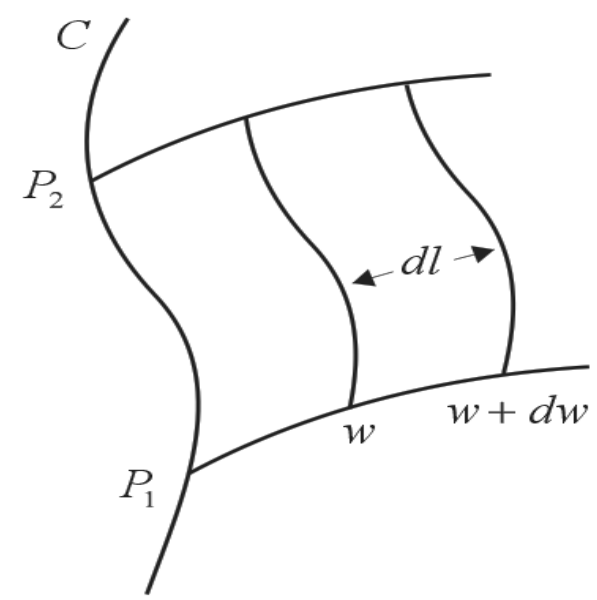

Fig. 3. Surface of constant retarded distance.

But when building the shell formed by $w, w+d w$ and the null cones at $P_{1}$ and $P_{2}$, we get for its 4-volume $d^{4} x=d l d \sigma=\left|w_{; a} w^{; a}\right|^{-1 / 2} \cdot d w \cdot d \sigma$, and after comparison with (15) implies that $\left|w_{; a} w^{; a}\right|^{-1 / 2} d \sigma=g^{-1 / 2}(P) \cdot \Delta^{-1} w^{2} d s d \gamma$, then (17) acquires the following form:

$$
d \sigma_{r}=g^{-1 / 2}(P) \Delta^{-1} w^{2} w_{; r} d s d \gamma
$$

On the other hand, from (4) we deduce the expression:

$$
w_{; r}=\Omega_{i^{\prime} r} \lambda^{i^{\prime}}-w^{-1}\left(\Omega_{i^{\prime} j^{\prime}} \lambda^{i^{\prime}} \lambda^{j^{\prime}}+\Omega_{i^{\prime}} \frac{d}{d s} \lambda^{i^{\prime}}\right) \Omega_{r}=\hat{o}_{r}-w^{-1}(X+W) \Omega_{r},
$$

where we used the notation $\hat{o}_{r}=\Omega_{i^{\prime} r} \lambda^{i^{\prime}}, X=\Omega_{i^{\prime} j^{\prime}} \lambda^{i^{\prime}} \lambda^{j^{\prime}}, W=\Omega_{i^{\prime}} \frac{d}{d s} \lambda^{i^{\prime}}=\Omega_{i^{\prime}} \mu^{i^{\prime}}$.

The substitution of (19) into (18) provides the result (3.35) of [4]:

$$
d \sigma_{r}=g^{-1 / 2}(P) \Delta^{-1} w\left[w \hat{o}_{r}-(X+W) \Omega_{r}\right] d s d \gamma,
$$

which is the generalization to curved spaces of the result (10.6) in [1]. The deduction of (20) was simple thanks to the radiation coordinates. Nevertheless, the usefulness of $z^{r}$ goes far beyond that; in our opinion, its true importance lies on the analogies that we can establish with the Minkowski space-time, which will be seen more clearly in the next section.

\section{Radiation Tensors}

In a flat space we have the following radiative part of the Maxwell tensor corresponding to the Liénard-Wiechert retarded field [23]:

$$
\stackrel{T_{r s}}{R}=e^{\prime 2} w^{-4}\left(\mu^{2}-w^{-2} W^{2}\right) \xi_{r} \xi_{s}, \quad e^{\prime}=\frac{e}{4 \pi},
$$


with $\mu^{2}=\mu_{r} \mu^{r}, \quad \mu_{r}=\frac{d \lambda_{r}}{d s}, \quad w=-\xi_{r} \mu^{r}, \quad W=-\xi_{r} \lambda^{r}$, which satisfies:

$$
\begin{aligned}
& T_{r s} \xi^{s}=0, \\
& T_{r s}^{, s}=0 .
\end{aligned}
$$

A tensor field is said to be of the radiative type when it satisfies the properties (22) and (23). The continuity equation (23) is consequence of:

$$
\left(\mu^{2} w^{-4} \xi_{r} \xi_{s}\right)^{s}=0, \quad\left(w^{-6} W^{2} \xi_{r} \xi_{s}\right)^{s}=0,
$$

which in turn are particular cases of the identity:

$$
\left[f\left(\mu^{2}\right) w^{-n} W^{m} \xi_{r} \xi_{s}\right]^{s}=0, \quad-n-m=-4,
$$

$f$ being an arbitrary function of $\mu^{2}$. It seems natural to wonder whether (21) can be extended to the curved space. The answer is positive under the two following prescriptions:

a).- Identify $\xi_{r}$ with $-\Omega_{r}$, see (4).

b).- Multiply (21) by $\left|J\left(\frac{z^{a}}{x^{b}}\right)\right|=g^{1 / 2}(P) \Delta$ due to the fact that $d^{4} x$ contains the factor $g^{-1 / 2}(P) \Delta^{-1}$ with respect to the corresponding expression for the flat space, see (16).

Thus

$$
T_{r s}=e^{\prime 2} g^{1 / 2}(P) \Delta w^{-4}\left(\mu^{2}-w^{-2} W^{2}\right) \Omega_{r} \Omega_{s}
$$

satisfies (23) with covariant derivative, due to the fact that the validity of (22) turns out to be evident. We can also expect the generalization of (24):

$$
\left[g^{1 / 2}(P) \Delta \mu^{2} W^{-4} \Omega_{r} \Omega_{s}\right]^{; s}=0, \quad\left[g^{1 / 2}(P) \Delta w^{-6} W^{2} \Omega_{r} \Omega_{s}\right]^{; s}=0,
$$

besides from (15) and (26) we have:

$$
T_{r s} d^{4} x=e^{\prime 2} w^{-2}\left(\mu^{2}-w^{-2} W^{2}\right) \xi_{r} \xi_{s} d s d w d \gamma
$$

which is important when performing some integrations around the world line of the charged particle. It is worth noting that (26) and (27) correspond to the results $(2.28, \ldots, 31)$ of Villarroel [6]. However, in our approach they can be obtained in a natural way by means of an explicit correspondence with the Minkowski space-time. The verification of (27) can be found in the work of the aforementioned author. 


\section{References}

[1] Synge JL (1970), Point-particles and energy tensors in special relativity. Ann. Mat. Pura Appl. 84: 33-60.

[2] Florides PS, McCrea J and Synge JL (1966), Radiation coordinates in general relativity. Proc. Roy. Soc. London A292: 1-13.

[3] Florides PS and Synge JL (1971), Coordinate conditions in a Riemannian space for coordinates based in a subspace. Proc. Roy. Soc. London A323: 1-10.

[4] Villarroel D (1975), Larmor formula in curved spaces. Phys. Rev. D11(10): 2733- 2739.

[5] DeWitt BS and Brehme RW (1960), Radiation damping in a gravitational field. Ann. of Phys. 9(2) : 220-259.

[6] Villarroel D (1975), Radiation from electron in curved spaces. Phys. Rev. D11(6) : 13831386.

[7] Ruse HS (1931), Taylor's theorem in the tensor calculus, Proc. London Math. Soc. 32(1): 87-92.

[8] Synge JL (1960), Optical observations in general relativity. Rend. Sem. Mat. Fis. Milano 30:1-35.

[9] Synge JL (1962), Relativity based on chronometry, in 'Recent developments in general relativity'. Pergamon Press: 441-448.

[10] DeWitt BS (1967), Quantum theory of gravity.III. Applications of the covariant theory. Phys. Rev. 162(5): 1239-1256.

[11] Buchdahl HA (1970), Point characteristics of some static spherically symmetric space times. Optica Acta 17: 707-713.

[12] Lathrop JD (1973), Covariant description of motion in general relativity. Ann. of Phys. 79(2): 580-595.

[13] Lathrop JD (1975), On Synge's covariant laws for general relativity. Ann. of Phys. 95(2): 508-517.

[14] Synge JL (1976), Relativity: the general theory. North-Holland, Amsterdam.

[15] Buchdahl HA (1979), Perturbed characteristic functions: Applications to the linearized gravitational field. Aust. J. Phys. 32: 405-410.

[16] Buchdahl HA and Warner NP (1979), On the world function of the Schwarzschild field. Gen. Rel. Grav. 10(11): 911-923.

[17] Buchdahl HA and Warner NP (1980), On the world function of the Gödel metric. $J$. Phys. A13: 509-516.

[18] Buchdahl HA (1985), Perturbed characteristic functions. II: second-order perturbation. Int. J. Theor. Phys. 24: 457-465.

[19] Roberts M (1993), The world function in Robertson-Walker spacetime. Astro. Lett. Comm. 28: 349-357.

[20] López-Bonilla J, Morales J and Rosales MA (1994), The Lorentz-Dirac equation in curved spaces. Pramana J. Phys. 43(4): 273-278.

[21] Sinaceur MA (1990), Dedekind et le programme de Riemann. Rev. Hist. Sci. 43: 221-294.

[22] Laugwitz D (2008), Bernhard Riemann 1826-1866. Turning points in the conception of mathematics. Birkhäuser, Boston, USA.

[23] Gaftoi V, López-Bonilla J and Ovando G (2001), Eigenvectors of the Faraday tensor of a point charge in arbitrary motion. Can. J. Phys. 79(1) : 75-80. 